Pulsar Astronomy -...2000 and Beyond

ASP Conference Series, Vol. 202, 2000

$M$. Kramer, N. Wex, and R. Wielebinski, cds.

\title{
OPTIMA \\ An Optical Pulsar Timing Analyser
}

\author{
Christian Straubmeier, Gottfried Kanbach \\ Max-Planck-Institut fuer extraterrestrische Physik, Giessenbachstrasse, \\ 85740 Garching, Germany
}

\begin{abstract}
OPTIMA is a small and mobile highspeed photometer which is primarily intended for the observation of young high energy pulsars at optical wavelengths. The detector system consists of a fiber fed photon counter based on avalanche photodiodes, a GPS timing receiver and an integrating CCD camera to ensure the correct positioning of the targeted object. In January 1999, OPTIMA proved its functionality by measuring the lightcurve of the Crab pulsar.
\end{abstract}

\section{Introduction}

Considering the whole spectrum of electro-magnetical waves, the low number of indisputable identifications of pulars in the optical band is striking, and up to now the search for optical variations at the pulsars' rotational frequencies has only succeeded in three significant and two suspected lightcurves. Nevertheless, the optical emission from the neutron star's surface or magnetosphere can provide important insights into the emission processes and thermal condition of pulsars. In the magnetosphere it marks the onset of the nonthermal high energy processes that extent to $\mathrm{X}$ - and $\gamma$-ray frequencies, and as the Rayleigh Jeans part of a thermal spectrum, it provides estimates on the size of the star and its equation of state.

To gain more infomation on pulsar emission in this decisive wavelength range, the $\gamma$-ray group of the Max-Planck-Institut fuer extraterrestrische Physik decided to build a new highspeed photometer, called OPTIMA, for use at ground-based telescopes.

\section{Principle of Operation}

The basic principle of operation is called photon-counting, where the arrivaltime of every single photon is recorded separately with a precision of only a few microseconds. As the optical flux from most of the targeted objects is too low to do a successful frequency analysis, the well known pulsar period from other wavelength bands is used for the essential folding to achieve a correct lightcurve histogram. Nevertheless, considering sources with a sufficiently high flux-level, it is also possible to search for pulsations at unknown frequencies, occultations of binary systems or quasi-periodic-oscillations. 


\section{Detector-System}

The basic design of the distributed detector system of OPTIMA consists of three major parts:

- Highspeed APD-Counter

To minimize the contribution of the background sky, the optical flux of the target is picked up at the focal-plane of the telescope by the use of a hexagonal bundle of seven fibers, whose diameters are chosen to match the expected seeing conditions and focal scale of the telescope. On the other side of each fiber, the photons are fed to dedicated avalanche photodiodes, which, in comparison to a photomultiplier tube, have a broadband response in the visible wavelength range and an outstanding quantum efficiency of up to $80 \%$.

To reduce the electronic noise and ensure a time-stable operation, the photodiodes and their preamplifiers are cooled and temperature-controlled by a multistage Peltier system to about $-30^{\circ} \mathrm{C}$.

- CCD-Guider

Apart from the flux of the targeted object, which is forwarded from the focalplane of the telescope to the highspeed counter, the light of the surrounding sky is reflected by a slanted mirror into a focal-reducer and afterwards detected by a CCD camera.

Thus, the known positions of stellar objects in the vicinity of the target, which might even be too faint to be visible by itself within reasonable integration times, can be used in conjunction with the CCD image to verify the positioning of the target on the fiber feed-through. Providing the necessary software and connection to the telescope control system, this unit can even generate suitable signals to represent an auto-guider and ensure a time-stable pointing.

- Timing and Controlling Unit

The timing and controlling unit is located at the observer's room and combines the photon detections of the highspeed APD-counter with the accurate timingsignals of a GPS-receiver. Moreover, most of the operating parameters of the OPTIMA detector-system are controlled from this unit and can be monitored and changed interactively.

\section{First Light}

In January 1999, a first version of the OPTIMA detector system has been successfully used at the 3.5 meter telescope at Calar Alto, Spain. As can be seen in the figure on the right, the basic performance of the acquisition, timing and analysing system could be demonstrated by the prompt recording of the well known lightcurve of the Crab pulsar.

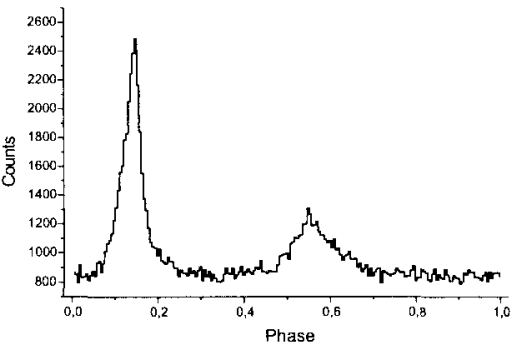

Figure 1. First Light 